\title{
Primary Immunodeficiencies
}

\author{
Michael Albert, Arjan Lankester, \\ and Andrew Gennery
}

\subsection{Introduction}

Primary immunodeficiency (PID) diseases arise from genetic defects that lead to abnormalities in immune cell development or function with a wide spectrum in severity and clinical manifestations. A subgroup of patients with an immunodeficiency present as a medical emergency which is associated with a chronic disabling and life-threatening clinical course. In these cases, allo-HSCT provides a life-saving and curative treatment modality. Replacement of the defective cell lineage by HSCT from healthy allogeneic donors remains the curative approach for these patients. Other management options including enzyme replacement therapy, gene transfer into autologous hematopoietic stem cells, and targeted therapies (see below) may provide an alternative approach to HSCT in specific immune deficiencies.

M. Albert

Pediatric SCT Program, Dr. von Hauner University Children's Hospital, Ludwig-Maximilians Universität, Munich, Germany

A. Lankester

Department of Pediatrics, Stem Cell Transplantation Program, Willem-Alexander Children's Hospital, Leiden University Medical Center, Leiden, The Netherlands

A. Gennery $(\bowtie)$

Paediatric Immunology + HSCT, Great North Children's Hospital, Newcastle University, Newcastle upon Tyne, UK e-mail: andrew.gennery@newcastle.ac.uk

\subsection{Diseases}

For HSCT purposes and thus for this handbook, PID may be broadly categorized into severe combined immunodeficiencies (SCID) and nonSCID. To further subdivide non-SCID, the phenotypic classification as suggested by the International Union of Immunological Societies (IUIS) Inborn Errors of Immunity Committee can be used, which encompasses $>300$ genetic causes of PID (Table 89.1).

Overall guidelines for HSCT for SCID and non-SCID diseases together with detailed protocols have been produced by the EBMT Inborn Errors Working Party (EBMT IEWP) and can be found online at https://www.ebmt.org/sites/ default/files/migration_legacy_files/document/ Inborn\% 20Errors\%20Working\%20Party\%20 ESID \%20EBMT\%20HSCT\%20Guidelines\%20

Table 89.1 Phenotypic classification of PID as suggested by the International Union of Immunological Societies (IUIS) Inborn Errors of Immunity Committee (Picard et al. 2018)
1. Combined immunodeficiency (CID)
2. CID with associated or syndromic features
3. Predominantly antibody deficiencies
4. Diseases of immune dysregulation
5. Congenital defects of phagocyte number, function, or both
6. Defects in intrinsic and innate immunity
7. Auto-inflammatory disorders
8. Complement deficiencies
9. Phenocopies of PID 
2017.pdf. An update of these guidelines is planned for 2018 based on current IEWP studies.

\subsection{SCID}

The overall frequency of SCID was for a long time estimated to be 1 in 50,000-100,000 live births. However, in recent years newborn screening programs making use of the T-cell receptor excision circles (TREC) technology have demonstrated that the frequency may actually be two- or more-fold higher with clear geographical and ethnic differences (Kwan et al. 2014; Rechavi et al. 2017).

The immunological phenotypes of SCID are shown in Table 89.2 representing monogenic inherited defects in T-, B-, and NK-cell differentiation leading to the absence or inactivity of corresponding mature cells. Over the past two decades, the genetic basis of an increasing number of SCID variants has been identified (Table 89.2) leading to modifications in transplant strategy dependent on the underlying defect particularly in SCID variants caused by defects in DNA repair genes.

In the absence of newborn screening programs, most patients present within the first

Table 89.2 Gene defects typically associated with specific SCID phenotypes

\begin{tabular}{|c|c|c|c|}
\hline $\mathrm{T}-\mathrm{B}+\mathrm{NK}-$ & $\mathrm{T}-\mathrm{B}+\mathrm{NK}+$ & T-B-NK- & T-B-NK+ \\
\hline $\begin{array}{l}I L 2 R G \\
(\mathrm{SCID}-\mathrm{X} 1)\end{array}$ & $I L 7 R$ & $A D A$ & LIG4 \\
\hline \multirow[t]{6}{*}{$J A K 3$} & $C D 3 D$ & $\begin{array}{l}A K 2 \\
\text { (reticular } \\
\text { dysgenesis }\end{array}$ & $R A G 1$ \\
\hline & $C D 3 E$ & & $R A G 2$ \\
\hline & $\begin{array}{l}C D 247 \\
(\mathrm{CD} 3 \zeta)\end{array}$ & & $\begin{array}{l}\text { DCLRE1C } \\
\text { (Artemis } \\
\text { def.) }\end{array}$ \\
\hline & COROIA & & $\begin{array}{l}\text { NHEJ1 } \\
\text { (Cernunnos } \\
\text { XLF) }\end{array}$ \\
\hline & $\begin{array}{l}\text { PTPRC } \\
\text { (CD45 } \\
\text { def.) }\end{array}$ & & $\begin{array}{l}\text { PRKDC } \\
\text { (DNA-PKcs } \\
\text { def.) }\end{array}$ \\
\hline & FOXN1 & & \\
\hline
\end{tabular}

Adapted from Picard et al. (2018)
3-6 months with unusually severe and recurrent infections or with opportunistic infections, the most common being Pneumocystis jiroveci pneumonia. Other common symptoms include diarrhea, dermatitis, and failure to thrive. Survival in SCID patients depends on expeditious T-cell reconstitution, and in the absence of successful HSCT, or in selected cases autologous stem cell gene therapy, most children die usually during the first year of life from overwhelming infection. It is recognized that as many as $50 \%$ of SCID patients are engrafted with maternal T-cells but in most instances these cells do not initiate GvHD. Transfusion-associated GvHD, on the other hand, is frequently lethal in SCID, and any patient with a possible diagnosis of SCID should receive irradiated blood products. Bacille Calmette-Guérin (BCG) vaccination can give rise to disseminated BCG-osis in SCID patients and should be avoided at birth if there is any suspicion or family history of immunodeficiency.

\subsubsection{General Principles in Allo- HSCT for SCID}

The Stem CEll Transplant for primary Immune Deficiencies in Europe (SCETIDE) registry has now collected data on SCID transplants comprising 50 years of HSCT experience, and a number of important publications have documented the outcomes and important risk factors (Fischer et al. 1990; Antoine et al. 2003; Gennery et al. 2010). Recently, studies from the North American group have reported similar findings (Pai et al. 2014; Heimall et al. 2017). The major factors influencing outcome reported in these studies include:

1. Preceding comorbidity (particularly infectious complications at HSCT) adversely affecting outcome

2. The type of donor with matched sibling donors having the best outcome ${ }^{1}$

${ }^{1}$ Recent data show that the type of donor and the immunological SCID phenotype have an ever-diminishing influence on outcome. 
3. The type of SCID, with T-B- forms of SCID having an inferior outcome (see footnote 1)

4. Age at transplant with patients $<3.5$ months having a favorable outcome

\subsubsection{Matched Sibling Donor HSCT for SCID}

During the last decade, the overall survival for MSD HSCT in SCID has improved to over 90\%. Somewhat remarkably, sibling donor BM may be infused into SCID recipients without the requirement for conditioning or GvHD prophylaxis. Infusion of sibling BM leads to the rapid development of T- and B-cell function post-HSCT, although usually only T-cells of donor origin develop and myeloid and erythroid cells remain of recipient origin. In T-/B+ SCID, the majority of patients achieve humoral reconstitution despite lack of donor B-cells, whereas following unconditioned HSCT in T-/B- SCID immunoglobulin dependence often persists.

\subsubsection{Other Matched Family and URD HSCT for SCID}

Overall survival rate following phenotypically matched related as well as URD transplants has steadily improved and is approaching MSD results (Gennery et al. 2010; Pai et al. 2014; Heimall et al. 2017). It is generally considered that the risk of rejection and particularly GvHD is too high for simple infusion of phenotypically matched marrow into SCID patients, so conditioning/GvHD prophylaxis is recommended. In a recent transatlantic study, survival rate in unconditioned URD HSCT was comparable with MSD HSCT, however at the expense of increased acute GvHD and inferior B-cellular immune reconstitution (Dvorak et al. 2014). A variety of conditioning regimes have been used, and current IEWP recommendations include the use of an IV BU/FLU- or TREO/ FLU-based protocol (details at http://www.ebmt. org/5WorkingParties/IEWP/wparties-ie5.html). Comparison of survival rates and immune function with these regimens is part of ongoing studies.

\subsubsection{HLA-Mismatched Family Donor for SCID}

Virtually all children have a haploidentical parental donor, and this is an alternative option especially as the donor is readily available. HLA disparity necessitates rigorous in vitro or in vivo TCD in order to avoid GvHD. Using mobilized PBSC as a preferred stem cell source, most centers employ either CD34-positive selection or CD3-/CD19-negative depletion methods to achieve a 4-5 $\log$ TCD achieving a threshold of $1-5 \times 10^{4} / \mathrm{kg} \mathrm{CD} 3^{+}$cells, below which GvHD prophylaxis is not required.

More recently, alternative haploidentical procedures including TCR alpha/beta depletion (Balashov et al. 2015; Shah et al. 2018) and PT-CY have emerged as HSCT options. Although promising survival rates have been reported, longer follow-up in a larger cohort of patients is required to determine the position of these approaches.

Some centers advocate performing transplants without the use of any conditioning, and survival rates of over $80 \%$ have been reported (Dvorak et al. 2014). However, the best results are seen in those transplanted at $<3.5$ months of age and in the absence of active infections. Despite general improvements in survival rate, the best results are still seen in the $\mathrm{TB}^{+}$subgroup of SCID patients. Even in these cases, B-cell function is only restored in the minority of patients. Conditioning regimes can be used to improve outcome, but the use of MAC regimes in children often $<1$ year of age is associated with significant comorbidity and leads to survival figures of $50-60 \%$. Individualized approaches making use of therapeutic drug monitoring or antibody-based conditioning strategies may provide novel and less toxic options to improve HSCT outcome in these vulnerable young infants.

\subsubsection{Unrelated CBT for SCID}

During the last decade, the availability of CBU plus the increased level of HLA matching degree has made $\mathrm{CB}$ a suitable alternative source of stem 
cells (Fernandes et al. 2012). There are some theoretical advantages for the use of cord blood stem cells for SCID, namely, rapid availability, as with haplotype-matched parental donors but with no requirement for TCD; less risk of GvHD compared to adult URD; no medical risk to the donor; and a greater proliferative life span which might be particularly important in such young recipients. Moreover, the usual limitation of cell dose in CBT is usually no issue in infants with SCID. There are also some specific disadvantages including slower engraftment, lack of viralspecific cytotoxic T-cells, and lack of availability of the donor for a boost HSCT.

\subsubsection{Omenn's Syndrome}

Omenn's syndrome (OS) is characterized by SCID typically associated with the triad of erythroderma, hepatosplenomegaly, and lymphadenopathy. There is a marked eosinophilia and a variable number of autologous, activated, and oligoclonal $\mathrm{T}$ lymphocytes (leaky SCID/CID), which infiltrate target organs and are generally poorly responsive to mitogens. Whereas outcomes in HSCT for OS were traditionally more difficult compared the classical SCID, results have improved in recent years (Gennery et al. 2010; Heimall et al. 2017). The overall mortality in these studies was lower than previously reported and was due to early recognition of OS and rapid initiation of treatment with topical/systemic immune suppression with steroids and/or cyclosporin A to control immune dysreactivity before proceeding to HSCT.

\subsubsection{HSCT for Radiosensitive SCID}

Patients with T-B- SCID due to radiosensitive disorders such as DNA ligase 4 deficiency, Cernunnos deficiency, DNA-PKcs deficiency are increasingly being identified and being considered for HSCT. As many of the conditioning regimens are particularly damaging to DNA, less toxic regimens are required to successfully treat these patients (Slack et al. 2018). No definitive studies are available, but a low-dose FLU/CY regime has been suggested by the EBMT IEWP (http://www.ebmt.org/5WorkingParties/IEWP/ wparties-ie5.html).

\subsection{Non-SCID Immunodeficiency}

From a HSCT viewpoint, the major difference with non-SCID patients in comparison with SCID patients is the requirement for a conditioning regimen to achieve engraftment. It is the goal to establish sufficient long-term donor chimerism in the affected cell lineage. The required degree of donor chimerism for full disease correction varies depending on PID and has not been established for all entities.

Many children with non-SCID PID have significant comorbidities at the time of HSCT. Conventional MAC preparation with BU-/ CY-based regimes has historically been associated with significant treatment-related toxicity and TRM. The Inborn Errors Working Party of EBMT has therefore in 2005 published detailed recommendations for conditioning and PID as discussed above. These recommendations include:

1. Replacement of CY with FLU, as the combination of BU/FLU appears to be better tolerated in these patients

2. Adding the option to replace BU with a structural analogue, TREO, which is similarly immuno- and myelosuppressive but causes less hepatic SOS/VOD (Slatter et al. 2018)

3. Establishing RIC to achieve stable engraftment of immunocompetent donor cells with reduced procedure-related morbidity and mortality (Veys 2010)

The latest outcome data for HSCT in nonSCID patients come from Europe (Gennery et al. 2010). In the 2000-2005 period, HSCT using an URD $(n=124)$ gave a 3 -year survival rate similar to a genoidentical donor ( $n=73$ ), $79 \%$ for both. Survival was $76 \%$ in phenoidentical transplants $(n=23)$ and worse in mismatched related donor transplants $(n=47,46 \%, p=0.016)$, in contrast 
to SCID patients (see above). Ten-year survival was significantly better for patients with WAS, phagocytic, and hemophagocytic disorders than for patients with T-lymphocyte immunodeficiencies $(71,63,58,47 \%$, respectively).

Unrelated CB donors appear to also give promising results in non-SCID immunodeficiency with 29/32 (91\%) patients surviving CBT matched for 4-6/6 HLA antigens.

The landscape of non-SCID PID and HSCT has dramatically changed over the last decade:

- HSCT outcomes have further improved with 80-90\% overall survival and low GVHD rates after MSD or MUD HSCT in almost every non-SCID PID entity (Güngör et al. 2014; Moratto et al. 2011).

- Haplo-HSCT has become a safe alternative, at least in the hands of experienced centers (Balashov et al. 2015; Shah et al. 2018).

- New genetic causes of PID are being described in accelerating frequency thanks to nextgeneration sequencing techniques.

- The concept of "pure" immunodeficiencies with predisposition to infections has been abandoned with newly described autoimmune, auto-inflammatory conditions or syndromal disorders with immunodeficiency. Many of these diseases can be cured by HSCT, while in syndromal disorders only the hematopoietic portion of the disease can be corrected, which may nevertheless be indicated and result in not just increased survival but also quality of life in selected patients.

- More PID patients are discovered with very mild or atypical phenotypes of well-known PID, and these often hypomorphic genetic variants are especially challenging with respect to timely recognition and management (Notarangelo et al. 2016).

- Especially in these "milder" cases, quality of life is increasingly a factor in HSCT decision making, especially compared to diseases with an immediate indication for HSCT such as SCID (Cole et al. 2013).

- The importance of DFS as compared to OS is increasingly appreciated and addressed in medium- to long-term outcome studies, also in comparison to non-HSCT approaches (Speckmann et al. 2017; Barzaghi et al. 2018).

- Adolescents and young adults with PID are appreciated as candidates for HSCT, and outcomes are encouragingly good (Albert et al. 2018; Fox et al. 2018).

- Patients with DNA double-strand repair disorders can safely undergo HSCT with irradiation-free, reduced-intensity regimens (Slack et al. 2018).

The consequence of all this has been that many more patients with PID are today considered for, referred for, and counselled about HSCT. On the other hand, it has made decision making with respect to HSCT much more difficult. A genetic diagnosis may make the decision to proceed to HSCT easier in patients with severe symptoms, but a genetic diagnosis alone should never be sufficient to indicate HSCT.

\subsection{Alternative Therapies}

Alternative treatments to HSCT have been developed for specific immunodeficiencies over the last three decades.

\subsubsection{Enzyme Replacement Therapy (ERT) for Adenosine Deaminase Deficiency (ADA-SCID)}

Enzyme replacement has been used in the treatment of ADA deficiency since 1987 (Chan et al. 2005). PEG-ADA is administered weekly or twice weekly by IM injection and leads to rapid metabolic correction with normalization of metabolic parameters which is then followed by cellular and humoral immune reconstitution. The extent of immune recovery is variable, and a significant number $(\sim 50 \%)$ remain on Ig replacement. Over a longer time period, patients show a decline in T-cell numbers and remain lymphopenic. Long-term follow-up shows that patients remain clinically well, but a number of cases of EBV-related lymphoma have been reported, sug- 
gesting decreased immune surveillance with time. Given the improved outcomes of HSCT in recent times and the availability of gene therapy, ERT may predominantly be considered a bridge to stem cell-based curative therapy.

\subsubsection{Gene Therapy for Specific Immune Deficiencies}

Autologous stem cell gene therapy (GT) via vector-mediated transfer of healthy copies of an affected gene into autologous $\mathrm{CD} 34^{+}$cells has progressed from a highly experimental therapy to the first licensed gene therapy for a PID (ADASCID) within the last two decades. One of the major advantages of GT is the elimination of the inherent risk of GVHD connected to any HSCT procedures.

Clinical trials performed with gamma retroviral vectors for ADA-SCID, X-linked SCID (SCID-X1), chronic granulomatous disease (CGD), and Wiskott-Aldrich syndrome (WAS) demonstrated that gene therapy can be an effective treatment option in patients lacking an HLA-identical donor (Hacein-Bey-Abina et al. 2002; Boztug et al. 2010; Stein et al. 2010; Aiuti et al. 2009). However, a high rate of insertional mutagenesis was observed in trials for SCID-X1, WAS, and CGD but not for ADASCID (Ott et al. 2006; Hacein-Bey-Abina et al. 2003; Braun et al. 2014). This has prompted the development of safer vectors based on selfinactivating retroviral or lentiviral vectors. Currently, a number of trials are ongoing or concluded for the diseases named above. All share the concept of submyeloablative or lymphodepleting conditioning followed by the infusion of auto-HSCT with added copies of the gene of interest. Promising results were published, especially for ADA-SCID (Cicalese et al. 2016), WAS (Aiuti et al. 2013) and SCID-X1 (Hacein-Bey-Abina et al. 2014). It is expected that gene editing approaches as alternative for gene addition technologies as currently employed will be developed in the next few years and may be employed to correct mature cells in diseases like CD40 ligand deficiency and IPEX Syndrome, as well as HSC.

In theory autologous stem cell gene therapy offers the appealing prospect of avoiding alloimmune reactions such as GVHD or rejection and a lower conditioning-related toxicity compared to allo-HSCT. But its exact role in treatment algorithms still needs to be defined in the absence of comparative studies. Also, logistic, regulatory, and economic hurdles still have to be overcome before its widespread application in the treatment of PID. Nevertheless, it has widened the therapeutic repertoire for patients with some PID. The rapid evolution of novel gene correction approaches promises to lead to even safer and more effective treatment options.

\subsubsection{Targeted Therapies}

The unravelling of new genetic PID entities, especially those caused by gain-of-function (GOF) variants and their pathophysiology, has for the first time opened the possibility to treat these diseases with highly specific, often small molecule inhibitors, some of which are already approved for other indications. These include but are not limited to abatacept for CTLA4 haploinsufficiency, ruxolitinib for STAT1 GOF, leniolisib for PIK3CD and PIK3R1, etanercept for ADA2 deficiency, and IL-1-targeted therapies (anakinra, rilonacept, and canakinumab) for auto-inflammatory recurrent fever syndromes (Jhamnani and Rosenzweig 2017; Ochs and Petroni 2018). At this point in time, the exact role of these agents in the treatment algorithm of PID is however unclear. Ideally, they could make HSCT unnecessary for some patients. On the other hand, concerns about long-term infection (and lymphoma) risk exist. In any case, in some patients with excessive autoimmunity and/or inflammation, these therapies can be viewed as an ideal bridge to HSCT and considered as a remission induction strategy to control the underlying PID, because they have the ability to bring the patient into the best possible clinical condition for HSCT. 


\section{Key Points}

- PIDs require a tailored approach to management, and transplant-specific protocols have been developed for these diseases.

- Preceding comorbidity, particularly infectious complications at HSCT and concurrent end-organ damage, adversely affects outcome; for many diseases, transplant at an early age is recommended.

- Alternative therapies instead of, or as a bridge to, transplant are increasingly available to improve patient outcomes.

\section{References}

Aiuti A, Cattaneo F, Galimberti S, et al. Gene therapy for immunodeficiency due to adenosine deaminase deficiency. N Engl J Med. 2009;360:447-58.

Aiuti A, Biasco L, Scaramuzza S, et al. Lentiviral hematopoietic stem cell gene therapy in patients with WiskottAldrich syndrome. Science. 2013;341:1233151.

Albert MH, Hauck F, Wiebking V, et al. Allogeneic stem cell transplantation in adolescents and young adults with primary immunodeficiencies. J Allergy Clin Immunol Pract. 2018;6:298-301.

Antoine C, Müller S, Cant A, European Group for Blood and Marrow Transplantation; European Society for Immunodeficiency, et al. Long-term survival and transplantation of haemopoietic stem cells for immunodeficiencies: report of the European experience 1968-99. Lancet. 2003;361:553-60.

Balashov D, Shcherbina A, Maschan M, et al. Singlecenter experience of unrelated and haploidentical stem cell transplantation with TCR $\alpha \beta$ and CD19 depletion in children with primary immunodeficiency syndromes. Biol Blood Marrow Transplant. 2015;21:1955-62.

Barzaghi F, Amaya Hernandez LC, Ambrosi A, et al. IPEX syndrome survivors following different therapeutic strategies: an international multicenter retrospective study. J Allergy Clin Immunol. 2018;141: 1036-49.

Boztug K, Schmidt M, Schwarzer A, et al. Stem-cell gene therapy for the Wiskott-Aldrich syndrome. N Engl J Med. 2010;363:1918-27.

Braun CJ, Boztug K, Paruzynski A, et al. Gene therapy for Wiskott-Aldrich syndrome-long-term efficacy and genotoxicity. Sci Transl Med. 2014;6:227ra33.

Chan B, Wara D, Bastian J, et al. Long-term efficacy of enzyme replacement therapy for adenosine deaminase (ADA)-deficient Severe Combined Immunodeficiency (SCID). Clin Immunol. 2005;117:133-43.
Cicalese MP, Ferrua F, Castagnaro L, et al. Update on the safety and efficacy of retroviral gene therapy for immunodeficiency due to adenosine deaminase deficiency. Blood. 2016;128:45-54.

Cole T, McKendrick F, Titman P, et al. Health related quality of life and emotional health in children with chronic granulomatous disease: a comparison of those managed conservatively with those that have undergone haematopoietic stem cell transplant. J Clin Immunol. 2013;33:8-13.

Dvorak CC, Hassan A, Slatter MA, et al. Comparison of outcomes of hematopoietic stem cell transplantation without chemotherapy conditioning by using matched sibling and unrelated donors for treatment of severe combined immunodeficiency. J Allergy Clin Immunol. 2014;134:935-943.e15.

Fernandes JF, Rocha V, Labopin M, et al. Transplantation in patients with SCID: mismatched related stem cells or unrelated cord blood? Blood. 2012;119:2949-55.

Fischer A, Landais P, Friedrich W, et al. European experience of bone-marrow transplantation for severe combined immunodeficiency. Lancet. 1990;336:850-4.

Fox TA, Chakraverty R, Burns S, et al. Successful outcome following allogeneic hematopoietic stem cell transplantation in adults with primary immunodeficiency. Blood. 2018;131:917-31.

Gennery AR, Slatter MA, Grandin L, Inborn Errors Working Party of the European Group for Blood and Marrow Transplantation, European Society for Immunodeficiency, et al. Transplantation of hematopoietic stem cells and long-term survival for primary immunodeficiencies in Europe: entering a new century, do we do better? J Allergy Clin Immunol. 2010;126:602-10.

Güngör T, Albert MH, Teira P, et al. Reduced-intensity conditioning and HLA-matched haemopoietic stemcell transplantation in patients with chronic granulomatous disease: a prospective multicentre study. Lancet. 2014;838:436-48.

Hacein-Bey-Abina S, Le Deist F, Carlier F, et al. Sustained correction of X-linked severe combined immunodeficiency by ex vivo gene therapy. N Engl J Med. 2002;346:1185-93.

Hacein-Bey-Abina S, von Kalle C, Schmidt M, et al. LMO2-associated clonal $\mathrm{T}$ cell proliferation in two patients after gene therapy for SCID-X1. Science. 2003;302:415-9.

Hacein-Bey-Abina S, Pai SY, Gaspar HB, et al. A modified $\gamma$-retrovirus vector for X-linked severe combined immunodeficiency. N Engl J Med. 2014;371:1407-17.

Heimall J, Logan BR, Cowan MJ, et al. Immune reconstitution and survival of 100 SCID patients posthematopoietic cell transplant: a PIDTC natural history study. Blood. 2017;130:2718-27.

Jhamnani RD, Rosenzweig SD. An update on gainof-function mutations in primary immunodeficiency diseases. Curr Opin Allergy Clin Immunol. 2017; 17:391-7.

Kwan A, Abraham RS, Currier R, et al. Newborn screening for severe combined immunodeficiency in 11 
screening programs in the United States. JAMA. 2014;312:729-38.

Moratto D, Giliani S, Bonfim C, et al. Long-term outcome and lineage-specific chimerism in 194 patients with Wiskott-Aldrich syndrome treated by hematopoietic cell transplantation in the period 19802009: an international collaborative study. Blood. 2011;118:1675-84.

Notarangelo LD, Kim MS, Walter JE, et al. Human RAG mutations: biochemistry and clinical implications. Nat Rev Immunol. 2016;16:234-46.

Ochs HD, Petroni D. From clinical observations and molecular dissection to novel therapeutic strategies for primary immunodeficiency disorders. Am J Med Genet A. 2018;176:784-803.

Ott MG, Schmidt M, Schwarzwaelder K, et al. Correction of X-linked chronic granulomatous disease by gene therapy, augmented by insertional activation of MDS1-EVI1, PRDM16 or SETBP1. Nat Med. 2006;12:401-9.

Pai S-Y, Logan BR, Griffith LM, et al. Transplantation outcomes for severe combined immunodeficiency, 2000-2009. N Engl J Med. 2014;371: 434-46.

Picard C, Bobby Gaspar H, Al-Herz W, et al. International Union of Immunological Societies: 2017 primary immunodeficiency diseases committee report on inborn errors of immunity. J Clin Immunol. 2018;38:96-128.

Rechavi E, Lev A, Simon AJ, et al. First Year of Israeli newborn screening for severe combined immunodeficiency-clinical achievements and insights. Front Immunol. 2017;8:1448.
Shah RM, Elfeky R, Nademi Z, et al. T-cell receptor $\alpha \beta+$ and $\mathrm{CD} 19+$ cell-depleted haploidentical and mismatched hematopoietic stem cell transplantation in primary immune deficiency. J Allergy Clin Immunol. 2018;141:1417-26.

Slack J, Albert MH, Balashov D, Inborn Errors Working Party of the European Society for Blood and Marrow Transplantation and the European Society for Immunodeficiencies, Stem Cell Transplant for Immunodeficiencies in Europe (SCETIDE), Center for International Blood and Marrow Transplant Research, Primary Immunodeficiency Treatment Consortium, et al. Outcome of haematopoietic stem cell transplantation for DNA-double strand breakage repair disorders. J Allergy Clin Immunol. 2018;141:322-8.

Slatter M, Rao K, Nademi Z, et al. Treosulfan and fludarabine conditioning for haematopoietic stem cell transplantation in children with Primary Immunodeficiency: UK experience. Biol Blood Marrow Transplant. 2018;24:529-36.

Speckmann C, Doerken S, Aiuti A, PCID-study of the Inborn Errors Working Party of the EBMT, et al. A prospective study on the natural history of patients with profound combined immunodeficiency (P-CID): a "first fifty" analysis. J Allergy Clin Immunol. 2017; 139:1302-10.

Stein S, Ott MG, Schultze-Strasser S, et al. Genomic instability and myelodysplasia with monosomy 7 consequent to EVI1 activation after gene therapy for chronic granulomatous disease. Nat Med. 2010;16:198-204.

Veys P. Reduced intensity transplantation for primary immunodeficiency disorders. Immunol Allergy Clin N Am. 2010;30:103-24.

Open Access This chapter is licensed under the terms of the Creative Commons Attribution 4.0 International License (http://creativecommons.org/licenses/by/4.0/), which permits use, sharing, adaptation, distribution and reproduction in any medium or format, as long as you give appropriate credit to the original author(s) and the source, provide a link to the Creative Commons license and indicate if changes were made.

The images or other third party material in this chapter are included in the chapter's Creative Commons license, unless indicated otherwise in a credit line to the material. If material is not included in the chapter's Creative Commons license and your intended use is not permitted by statutory regulation or exceeds the permitted use, you will need to obtain permission directly from the copyright holder.

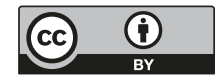

